\title{
Une action collective analysée par ses acteurs : une association d'irrigants en Tunisie
}

\author{
Azza Ben Mustapha ${ }^{1}$, Nicolas Faysse ${ }^{2}$, Serge Marlet $^{3}$, Jean-Yves Jamin ${ }^{4}$ \\ 1 Doctorante en sociologie, AgroParisTech (ABIES), 75005 Paris, France \\ 2 Socioéconomiste, CIRAD, UMR G-Eau, 34398 Montpellier, France \\ 3 Agronome, CIRAD, UMR G-Eau, 34398 Montpellier, France \\ 4 Agronome, CIRAD, UMR G-Eau, 34398 Montpellier, France
}

Les auteurs examinent un dispositif de recherche original mis en place auprès d'acteurs impliqués dans l'usage et la gestion d'un système collectif d'irrigation en Tunisie. Les grilles théoriques d'analyse de l'action collective par les sciences sociales sont utilisées pour accompagner ces acteurs dans une démarche d'autoanalyse. Cet exercice de réflexivité est l'objet d'un examen critique qui porte aussi bien sur le dispositif de recherche qu'il implique que sur les critères qui permettent de l'évaluer. Il pose plus généralement la question de la notion de "performance », retenue ici comme objet de la démarche réflexive.

La Rédaction

\section{Mots-clés :}

agriculture irriguée ; gouvernance ; action collective ; critères de performance ; perceptions d'acteurs ; Tunisie

\section{Keywords:} irrigated agriculture; governance; collective action; performance factors; actor viewpoints; Tunisia
Résumé - En Tunisie, les études sur les associations d'irrigants ont souvent diagnostiqué des performances médiocres, mais n'ont proposé que des analyses incomplètes des facteurs influençant ces performances. Dans la présente étude, les acteurs intervenant dans une association d'irrigants ont été invités à exprimer leurs points de vue sur les objectifs de l'association et les facteurs jouant sur ses performances, en réagissant à des exemples inspirés de trois théories de l'action collective et adaptés à leur situation. Ces acteurs sont les agriculteurs, le conseil d'administration, les salariés de l'association et les agents de l'administration publique. Les analyses différenciées faites par les acteurs ont permis une meilleure compréhension de leurs attentes vis-à-vis de l'association et des relations entre ces acteurs. Cet usage des théories de l'action collective s'est avéré être un outil d'élicitation innovant pour révéler les points de blocage et améliorer les performances de l'association.

\footnotetext{
Auteur correspondant : A. Ben Mustapha, azza.benmustapha@gmail.com
} 
En Tunisie, les politiques agricoles ont conféré, depuis 1992, un caractère stratégique à la création d'associations d'irrigants qui gèrent les périmètres publics irrigués sous le statut de groupement de développement agricole (GDA). Ces politiques conçues et mises en œuvre de façon descendante ont surtout servi pendant longtemps à renforcer le contrôle, au niveau local, du parti au pouvoir jusqu'à la Révolution du jasmin en 2011 (Canesse, 2010). Ce parti a ainsi joué un rôle important dans la désignation des élus aux conseils d'administration (Mouri et Marlet, 2005 ; Elloumi, 2011), qui lui servaient de sources d'information et de relais. Les GDA étaient ainsi des structures peu représentatives des agriculteurs adhérents (Elloumi et al., 2010), qui n'avaient à aucun moment été impliqués dans une réflexion sur les objectifs et les missions assignés à ces groupements.

Les études réalisées par les chercheurs et les décideurs ont dans leur ensemble considéré les performances de ces GDA comme médiocres (Al Atiri, 2007 ; Elloumi, 2011) pour diverses raisons. Romagny et Riaux (2007) ont mis en avant le manque de pouvoir décisionnel dévolu aux GDA et l'absence de réorganisation des relations entre l'État et les agriculteurs. Des chercheurs (Ben Salem et al., 2007 ; Elloumi, 2011) et des agents du ministère de l'Agriculture (Al Atiri, 2007) ont insisté sur leurs carences en matière de gestion technique et financière des périmètres irrigués. Ces constats n'ont cependant pas été intégrés dans un cadre d'analyse commun, et n'ont été posés que de manière générale au niveau de l'ensemble du pays, offrant un intérêt limité pour comprendre des situations spécifiques souvent complexes.

Dès le lendemain de la Révolution tunisienne, les membres des GDA ont contesté les conseils d'administration en place. Ils ont pointé leur manque de représentativité et leur mauvaise gestion, et ont affirmé leur volonté de choisir eux-mêmes leurs représentants (Gana, 2011). Ces réclamations ont débouché sur de nouvelles élections. Outre une amélioration du fonctionnement de la démocratie locale au sein de ces GDA, la Révolution a permis une plus grande liberté de parole (Elloumi, 2011; Gana, 2011). Les acteurs locaux ont acquis une plus grande capacité d'exprimer leurs points de vue et leurs propres analyses, et celle-ci peut désormais être mieux utilisée pour comprendre les situations complexes des GDA.

Le présent article ${ }^{1}$ a pour but d'expliciter les analyses que formulent les acteurs d'un groupement d'irrigants sur les objectifs et les performances de leur GDA. Les

\footnotetext{
1 L'article est fondé sur des travaux menés dans le cadre du projet Programme d'actions pilotes d'appui aux groupements de développement agricole dans les systèmes irrigués de Tunisie (Pap-Agir). Ce programme est financé par l'Agence française de développement, dans le cadre de de la seconde phase du Projet d'investissement sectoriel eau (Piseau 2).
}

acteurs considérés ici sont les agriculteurs, les élus au conseil d'administration, le personnel technique salarié du GDA et les agents du ministère de l'Agriculture chargés du suivi des GDA. L'étude des points de vue des différents acteurs sur les facteurs qui pourraient influencer les performances de leur groupement a été menée au regard des objectifs que chacun des acteurs assigne au GDA. Pour aider les acteurs à expliciter leurs analyses, nous avons fait le choix d'utiliser un certain nombre de facteurs de performance, tels que proposés par trois grilles d'analyse des théories de l'action collective issues des sciences sociales. Ces facteurs ont été, au préalable, adaptés aux situations locales au travers d'exemples concrets, construits à partir du quotidien des acteurs. Nous avons postulé que ces facteurs permettent de rendre comparables les différents points de vue et de structurer leurs analyses. Le choix des grilles d'analyse mobilisées a été effectué sur la base de leur clarté et de l'utilité opérationnelle des définitions qu'elles proposent des performances d'une organisation et des facteurs permettant de les améliorer.

Nous n'avons pas utilisé de définition préétablie de ce que sont les performances d'une association d'irrigants. Notre démarche a été fondée sur l'explicitation, par les acteurs, de la manière dont ils estimaient que le GDA devrait fonctionner et, en fonction de cela, de comment ils jugeaient qu'il fonctionnait. Au-delà de l'évaluation des performances du GDA, nous nous intéressons plus généralement à la façon dont les acteurs expriment leur analyse $\mathrm{du}$ fonctionnement de leur système et aux pistes qu'ils jugent pertinentes pour son amélioration.

\section{Cadre conceptuel}

Au cours des trois dernières décennies, de nombreux pays ont réorganisé leur secteur de l'irrigation. Un aspectclé de ces réformes, souvent promues par les bailleurs de fonds internationaux, a été de transférer la gestion des périmètres irrigués à des associations d'usagers (Johnson et al., 1995). Ce processus a été étudié pour comprendre le lien entre la démarche de transfert choisie et les performances de ces associations (Vermillon, 1997 ; Samad et Vermillion, 1999), et identifier les moyens d'améliorer ces performances (Bos et al., 1994). Meinzen-Dick et al. (2004) ont ainsi expliqué l'échec de plusieurs expériences de transfert de gestion de l'irrigation aux agriculteurs, par le manque d'attention accordée à la compréhension des conditions d'émergence et de maintien de l'action collective face aux problèmes rencontrés localement. D'autres chercheurs ont intégré le point de vue des acteurs sur l'évaluation de leurs actions collectives, à partir de réponses quantifiées, pour la gestion de problèmes environnementaux (Mermet et al., 2005 ; Beaurain et al., 2009) ou pour le secteur de l'irrigation (Bos et al., 1994; Shah, 1996). Ces études se sont cependant peu intéressées à 
l'analyse que les acteurs peuvent faire de leur propre action collective, que ce soit du point de vue de ses objectifs ou de ses critères de performance.

Or, selon Giddens (1984), la relation entre savoirs sociologiques et savoirs des acteurs devrait s'inscrire dans une "double herméneutique », c'est-à-dire un double processus où les chercheurs observent et interprètent des réalités déjà interprétées par les acteurs (lorsqu'ils sont les objets de la recherche), mais aussi où les concepts et résultats des sciences sociales sont euxmêmes compréhensibles et utilisables par ces mêmes acteurs. Nous nous proposons d'utiliser cette double réflexivité et avons donc choisi trois grilles d'analyse de l'action collective comme base de discussion avec les acteurs d'un groupement d'irrigants : celle élaborée par Ostrom $(1990$; 1992), celle de Shah $(1995 ; 1996)$ et celle proposée par Vermillon (1997) et Giordano et al. (2006). Ces trois grilles ne sont pas représentatives de l'ensemble des théories des sciences sociales portant sur l'action collective et $n^{\prime}$ ont pas été sélectionnées sur leur possible "véracité », positionnement ou représentativité par rapport à l'ensemble des théories existantes. Nous les avons retenues parce qu'elles ont été conçues à partir de l'analyse d'un grand nombre de situations et ont toutes considéré, partiellement ou spécifiquement, le cas des associations d'irrigants. Nous avons donc postulé que ces grilles contenaient des propositions à même de faire réagir nos interlocuteurs.

\section{Grille d'analyse d'Ostrom}

Ostrom fait partie du courant de l'économie institutionnelle. Ostrom (1990) a défini la performance d'une organisation comme la capacité à résoudre ce qu'elle a appelé "les situations de dilemmes sociaux», définies comme des moments de confrontation entre intérêts individuels et intérêts collectifs. Elle a identifié des principes communs aux règles de gestion durable des ressources naturelles et des biens en accès commun. À chaque principe peut être associé un facteur susceptible $d$ 'influencer la performance d'une action collective. Ces principes sont en lien avec, par exemple: la capacité des individus concernés à modifier les règles de gestion des ressources en accès commun, la mise en place de mécanismes de résolution de conflits, la reconnaissance par l'État des associations d'usagers et des règles de gestion que ces usagers ont conçues (Ostrom, 1990 ; 1992). Un principe énoncé par Ostrom (1990, p. 101) stipule en particulier que les autorités extérieures ne devraient pas affaiblir la capacité des usagers à concevoir leurs propres règles.

\section{Grille d'analyse de Shah}

Shah $(1995$; 1996) a étudié les conditions de succès et d'échec des coopératives d'agriculteurs. Il considère comme performante, toute coopérative qui accomplit ce que ses membres attendent d'elle, et ceci indépendamment de ce que peut en penser toute institution extérieure. Shah (1995) a montré que les coopératives performantes étaient souvent initiées par les agriculteurs, l'État venant alors seulement en accompagnement, voire n'intervenant pas du tout (capacité d'autocréation). Ces coopératives développent des formes d'adaptation face aux différentes contraintes qui menacent leur fonctionnement (capacité d'autopréservation), et améliorent leurs capacités pour atteindre le but pour lequel elles ont été créées (capacité d'autoamélioration). Les coopératives performantes cherchent à occuper une place centrale dans le quotidien de leurs membres en élargissant souvent la gamme des services proposés, pour mieux répondre à leurs besoins. Shah insiste sur deux facteurs de performance : la capacité des agriculteurs à peser sur les décisions du conseil d'administration et à lui demander des comptes, et la capacité du conseil d'administration à contrôler les activités du personnel technique.

\section{Grille d'analyse de Vermillon et Giordano}

À l'International Water Management Institute (IWMI), Vermillon (1997) puis Giordano et al. (2006) se sont intéressés au processus de transfert de la gestion de l'irrigation en étudiant le lien entre la démarche de transfert et la performance de la gestion qui en résulte. Selon eux, la performance des associations d'irrigants est essentiellement définie par une bonne gestion technique et financière, et en particulier une bonne qualité du service de distribution de l'eau. Les principaux facteurs de performance qu'ils proposent sont : l'autonomie dans la gestion du service de l'eau, la liberté d'appliquer les sanctions choisies localement, et le fait de tirer un profit suffisant de l'irrigation (ce dernier facteur est aussi une idée que reprendront Shah et al., 2002). Vermillion (1997) et Giordano et al. (2006) ont donné une grande importance au cadre légal et réglementaire qui formalise les dispositifs de gestion et officialise les relations entre les différents acteurs.

Le tableau 1 résume quelques facteurs de performance issus des trois grilles d'analyse mobilisées, en précisant les auteurs qui les ont évoqués. Ils ont été répartis en un premier groupe qui concerne le niveau intraorganisationnel et un second groupe intervenant dans la relation entre l'association d'irrigants et l'administration locale. Notre démarche n'est pas de donner plus d'importance aux facteurs les plus cités par les théories mobilisées, mais de retenir des sujets susceptibles de faire réagir les acteurs.

\section{Le groupement étudié}

En Tunisie, les GDA délivrent de l'eau à leurs adhérents, et parfois aussi à des agriculteurs abonnés mais 
Tableau 1. Facteurs de performance de l'action collective selon plusieurs auteurs.

\begin{tabular}{|c|c|c|c|}
\hline \multirow[b]{2}{*}{ Facteurs de performance de l'action collective } & \multicolumn{3}{|c|}{ Auteurs } \\
\hline & Ostrom & Shah & $\begin{array}{l}\text { Vermillion et } \\
\text { Giordano et al. }\end{array}$ \\
\hline \multicolumn{4}{|l|}{ Relations internes à l'association } \\
\hline La capacité du leader à bien gérer l'association & $\mathrm{NE}$ & - & NE \\
\hline La capacité des membres à contrôler la gestion du conseil d'administration & \multirow{3}{*}{ NE } & \multirow{3}{*}{+} & \multirow{3}{*}{ NE } \\
\hline La capacité du conseil d'administration à répondre aux demandes des membres & & & \\
\hline L'implication des agriculteurs dans le fonctionnement du GDA & & & \\
\hline La possibilité pour les membres de modifier les règles opérationnelles & \multirow[b]{2}{*}{+} & \multirow[b]{2}{*}{+} & \multirow[b]{2}{*}{+} \\
\hline $\begin{array}{l}\text { La capacité des agriculteurs à conduire et contrôler par eux-mêmes la création } \\
\text { de leurs associations }\end{array}$ & & & \\
\hline $\begin{array}{l}\text { La capacité des agriculteurs à tirer suffisamment profit des activités agricoles } \\
\text { pour pouvoir financer la gestion et la maintenance de leur système irrigué }\end{array}$ & \multirow{2}{*}{ NE } & \multirow{2}{*}{+} & \multirow{2}{*}{+} \\
\hline $\begin{array}{l}\text { Le pilotage au quotidien par le conseil d'administration des activités du per- } \\
\text { sonnel technique }\end{array}$ & & & \\
\hline $\begin{array}{l}\text { Les sanctions en cas de non-respect par les membres des règles de fonctionne- } \\
\text { ment choisies par les membres }\end{array}$ & + & NE & + \\
\hline La possibilité pour l'association de modifier ses propres limites d'action & + & NE & NE \\
\hline \multicolumn{4}{|l|}{ Relation association - administration } \\
\hline $\begin{array}{l}\text { La non-intervention de l'administration dans le fonctionnement quotidien de } \\
\text { l'association }\end{array}$ & NE & + & NE \\
\hline La reconnaissance de l'administration des règles définies localement & + & NE & NE \\
\hline $\begin{array}{l}\text { L'existence d'un cadre formel écrit, clair et approuvé par les parties prenantes, } \\
\text { décrivant les règles de fonctionnement des relations entre l'association et } \\
\text { l'administration }\end{array}$ & NE & NE & + \\
\hline
\end{tabular}

non-adhérents (ils achètent de l'eau du GDA mais ne lui payent pas de cotisation annuelle). Les acteurs impliqués au quotidien dans la gestion du GDA sont le conseil d'administration (CA), élu par les adhérents, et un personnel technique composé d'agents salariés du GDA chargés de la gestion technique (exploitation et maintenance $\mathrm{du}$ réseau) et financière. Le GDA est principalement placé sous la tutelle de la représentation locale du ministère de l'Agriculture, appelée "commissariat régional de développement agricole »(CRDA), qui est chargé des fonctions d'appui et de contrôle. Le CRDA peut intervenir pour effectuer des actions de maintenance à la demande des GDA. Il gère aussi, lorsqu'il existe, le réseau d'amenée d'eau en amont du GDA. Un contrat de gérance, signé entre le CRDA et le GDA, décrit les engagements de chacun, notamment en termes de maintenance et, le cas échéant, les conditions de fourniture d'eau au GDA par le CRDA.

Le GDA étudié se trouve dans le gouvernorat de Sousse, à $140 \mathrm{~km}$ au sud de Tunis ${ }^{2}$. Une partie du périmètre irrigué était auparavant administrée par deux associations d'irrigants distinctes, gérant deux forages exploités depuis 1980. En 2003, un périmètre irrigué a été créé par l'État, en partie sur les terres de ces deux associations ; il est alimenté par les eaux d'un barrage situé dans le gouvernorat de Kairouan. En 2009 et 2011, à l'initiative de l'administration, le GDA créé par celle-ci pour la gestion du périmètre irrigué par les eaux du barrage a été fusionné avec les deux associations gérant les forages. L'eau du barrage est distribuée par une entreprise parapublique (la Secadenord), qui la vend au CRDA à 0,05 dinar tunisien $3 / \mathrm{m}^{3}$. Le CRDA la revend au GDA à $0,09 \mathrm{TND} / \mathrm{m}^{3}$, et ce dernier la distribue aux agriculteurs au tarif de $0,15 \mathrm{TND} / \mathrm{m}^{3}$.

Au début de chaque campagne agricole, et en fonction du niveau de l'eau dans le barrage, le Bureau de la planification et des équilibres hydrauliques ${ }^{4}$ décide des

2 Par souci d'anonymat, nous avons choisi de ne présenter que l'emplacement géographique du groupement.

31 dinar tunisien (TND) valait 0,48 euro au 31/12/2012.

4 Le Bureau de la planification et des équilibres hydrauliques est une structure rattachée au cabinet du ministre de l'Agriculture et des Ressources hydrauliques. 
quantités mensuelles allouées à tous les périmètres irrigués du gouvernorat de Sousse par la Secadenord. Le CRDA répartit ces quantités en fonction des superficies irrigables (estimées en se basant sur les superficies irriguées les campagnes précédentes).

Le périmètre étudié s'étend sur une superficie totale de 340 ha et comptait, pour la campagne agricole 20112012, 94 usagers, dont 47 adhérents au GDA. En 2012, la superficie irriguée était de 120 ha et était cultivée principalement en cultures maraîchères, en plein champ et sous serre. Les cultures irriguées sont pratiquées en intercalaire avec l'olivier, qui était déjà cultivé avant l'arrivée de l'irrigation.

Depuis la création du GDA, trois CA se sont succédé, élus en 2003, 2008 et 2011. Le premier CA était composé de notables de la zone, respectés par les membres du GDA. Les élections du deuxième CA (en 2008) se sont faites sous l'influence des représentants locaux du ministère de l'Intérieur, qui a voulu redynamiser le CA dans le sens des intérêts du parti alors au pouvoir, le Rassemblement constitutionnel démocratique (RCD) ; le président alors élu à la tête du CA et sa famille n'ont par la suite pas payé une part de leur consommation en eau, et ont ainsi contribué à l'accumulation de dettes importantes du GDA envers le CRDA (environ 30000 TND). Les agriculteurs ont essayé de limoger ce président en 2010, mais ont échoué, car les autorités locales se sont opposées à la tenue de l'assemblée générale extraordinaire nécessaire à une telle révocation. Après la Révolution, la première action des adhérents a été de limoger le président en organisant pour cela des élections extraordinaires. Voulant rompre avec le passé, les agriculteurs ont choisi d'élire un agriculteur ayant une petite exploitation ( 0,5 ha d'arboriculture), qui exerçait le métier de médecin et qui n'avait que peu d'intérêts personnels à défendre du point de vue du GDA. Le trésorier fut maintenu, bien qu'il ait appartenu au RCD, parce qu'il était considéré comme un agriculteur expérimenté et que les autres s'adressaient souvent à lui pour des conseils.

\section{La démarche méthodologique}

La démarche a été mise en œuvre durant l'été 2012, en deux étapes : une première qualifiée d'exploratoire et une seconde de mise en débat. Durant la phase exploratoire, nous avons effectué plusieurs passages auprès du GDA, sur les parcelles de quelques irrigants et auprès de l'administration, afin que les acteurs puissent nous identifier et nous faire petit à petit confiance. Ensuite, des entretiens individuels ont été effectués, auprès de 21 agriculteurs non élus au CA (mais impliqués dans le GDA et motivés pour nous répondre), deux élus du CA (président et trésorier), deux salariés du personnel technique (aiguadier et directeur technique) et un agent du CRDA affecté à la cellule chargée du suivi des GDA. Les personnes contactées ont été invitées à exprimer leur point de vue sur l'histoire du périmètre, les objectifs du GDA, les principaux enjeux de gestion aussi bien techniques que sociaux, et les facteurs de performance du GDA. Cette première phase nous a permis de sélectionner, parmi les facteurs de performance présentés dans le tableau 1, ceux qui nous paraissaient correspondre le mieux aux enjeux de gestion tels qu'exprimés par les personnes interrogées.

Lors de la deuxième phase de mise en débat, nous avons associé à chacun des facteurs sélectionnés un exemple concret, déjà vécu ou qui pourrait avoir lieu dans le GDA étudié. Une seconde série d'entretiens individuels a été menée avec cinq agriculteurs parmi ceux rencontrés durant la première phase (choisis parce qu'ils nous étaient apparus comme étant particulièrement intéressés pour discuter de la gestion du GDA), le directeur technique, l'aiguadier, le trésorier, le président du groupement et le membre de la cellule de suivi des GDA au sein du CRDA déjà sollicité. Les exemples proposés ont été présentés à chaque personne interrogée, qui a été invitée à exprimer son point de vue (favorable, opposé, etc.) et à l'argumenter en explicitant ses prises de position. Souvent, les personnes interrogées ont ensuite généralisé, de leur propre initiative, leur analyse au-delà de l'exemple proposé.

\section{Résultats de la phase exploratoire}

\section{Principaux enjeux de gestion du GDA}

Les agriculteurs contactés partagent une même vision concernant l'objectif principal du GDA qui est, selon eux, d'assurer un bon service de l'eau aux usagers. Ils estiment que l'eau doit être disponible en quantité et en qualité, avec une flexibilité dans la desserte. Le CA et le personnel du GDA pensent que l'objectif du groupement est de fournir de l'eau à ses membres tout en assurant une situation financière stable. Le fait d'avoir mentionné l'importance de l'équilibre budgétaire de l'association renvoie aux difficultés auxquelles le groupement a fait face pendant la campagne agricole 20112012 et aux dettes accumulées par l'ancien président. Pour l'agent CRDA de la cellule de suivi des GDA, l'objectif du groupement est de distribuer aux agriculteurs l'eau que le CRDA lui fournit et d'assurer la maintenance des équipements hydrauliques mis à sa disposition par l'État. Il donne une définition détaillée des bonnes performances d'un GDA en se basant sur des paramètres quantifiables de types administratif, financier et technique. 
L'eau délivrée est considérée par les agriculteurs, le personnel technique et le CA comme de mauvaise qualité, du fait de la turbidité de l'eau provenant du barrage (la principale ressource alimentant le GDA) et de la qualité insuffisante des équipements de filtration. Aucun engagement concernant la qualité de l'eau n'est mentionné dans le contrat de gérance CRDA-GDA, dont les agriculteurs, le président et le trésorier ne connaissent d'ailleurs pas le contenu. Le colmatage progressif des filtres provoque une baisse sensible de la pression, en particulier parce que le GDA n'assure pas régulièrement leur maintenance. De plus, le GDA ne dispose pas de moyens financiers permettant de les remplacer.

Le personnel technique du GDA applique un tour d'eau flexible pour mieux répondre à la demande des usagers. Cela se pratique contre l'avis du CA, qui souhaiterait un tour d'eau planifié. L'aiguadier est très influent dans la gestion, et ce d'autant plus que le CA est peu présent dans le fonctionnement quotidien. Les activités de l'aiguadier ne se limitent pas à l'ouverture et la fermeture des vannes, il est aussi l'intermédiaire entre les agriculteurs et le directeur technique du GDA pour la planification du tour d'eau et le paiement des redevances. L'aiguadier joue aussi parfois un rôle dans la vulgarisation et dans l'entretien des bornes d'irrigation pour le compte des agriculteurs. Des agriculteurs situés hors du périmètre demandent souvent à recevoir de l'eau ; le CA accepte parfois, car cela permet d'améliorer la situation financière du GDA. Cependant, le CRDA se déclare opposé à ces "extensions ", arguant qu'il s'agit $\mathrm{d}^{\prime}$ une prise de risque importante si, pour une raison ou pour une autre, le GDA reçoit moins d'eau que prévu durant la campagne (ce qui est arrivé durant l'été 2013). Cette opposition est symbolique de l'autonomie relative du GDA par rapport au CRDA : le GDA ne mobilise pas lui-même sa principale ressource en eau, il n'est qu'un intermédiaire dans sa distribution.

Bien que l'objectif principal du GDA diffère selon les acteurs interrogés, tous s'accordent pour considérer que sa gestion est médiocre. La cause citée par les acteurs de prime abord est la mauvaise gestion des anciens membres du CA. Les agriculteurs citent aussi le manque de discipline et de savoir-faire du personnel technique, ainsi que sa faible volonté de servir l'intérêt du GDA. Pour le président actuel, la mauvaise gestion du GDA par l'ancien président a aussi été permise par un manque d'implication dans cette gestion du personnel technique et des élus du CA. Pour améliorer cette situation, les agriculteurs mettent l'accent sur l'importance de la qualité de la relation entre les agriculteurs, le CA et le personnel technique, et expriment le besoin d'être encadrés, suivis et écoutés. Certains pensent que des facteurs externes (tels que la baisse du débit d'eau fourni par le CRDA) peuvent aussi affecter les performances du GDA. Les agriculteurs seraient aussi intéressés par le fait que le
GDA (qui en 2012 ne faisait que distribuer de l'eau) propose d'autres services, tels que la location d'équipements agricoles, le conseil technique et l'aide à la commercialisation.

\section{Construction des facteurs de performance et des exemples mis en discussion avec les acteurs}

À partir des enjeux et problèmes décrits par les acteurs, ainsi que des possibilités d'amélioration des performances qu'ils ont présentées, six thématiques principales liées aux performances du GDA étudié ont été sélectionnées. Le tableau 2 présente les facteurs sélectionnés (première colonne de gauche) à partir de la liste proposée dans le tableau 1, ainsi que les exemples choisis, mis en discussion avec les acteurs locaux (colonne du milieu). Ces exemples représentent soit des facteurs généraux, mais aisément compréhensibles par l'ensemble des acteurs, soit des actions concrètes susceptibles d'améliorer les performances du GDA. En ce qui concerne la diversification des services fournis par le GDA, nous avons choisi de faire réagir les acteurs sur la possibilité d'acheter un tracteur en commun au sein du groupement, un service mentionné à plusieurs reprises lors des entretiens de la phase exploratoire. La participation des agriculteurs à la gestion quotidienne a été illustrée à travers l'hypothèse d'une participation des agriculteurs à l'entretien des filtres. La question de l'autonomie du groupement par rapport à l'administration, notamment dans sa capacité à modifier les limites de sa zone d'action, a été mise en discussion à travers la possibilité pour le GDA de fournir de l'eau hors du périmètre, et la manière dont il en rend compte au CRDA. La question du contrôle de la gestion du CA par les membres a été mise en discussion plus particulièrement en proposant aux personnes interrogées d'expliquer l'absence de réactions des différents acteurs impliqués face aux dettes accumulées par l'ancien président et sa famille.

\section{Résultats de la phase de mise en débat}

\section{Contrôle de la gestion par le CA : la question de la dette}

L'endettement du groupement vis-à-vis du CRDA est lui-même causé par celui des agriculteurs envers le GDA. Les entretiens menés avec les agriculteurs montrent leur manque de connaissance de la situation financière réelle $\mathrm{du}$ groupement. Les agriculteurs ont aussi évoqué la complicité entre les membres du précédent CA (qui a été remplacé en 2011 et qui n'était plus en place lors de l'enquête) et le personnel technique dans l'accumulation des dettes. Le personnel technique met, quant à lui, l'accent sur la proximité qu'il y avait, avant la Révolution, entre le gouverneur de la région et l'ancien CA. 
Tableau 2. Facteurs de performance, exemples associés mis en discussion avec les acteurs, et points de vue sur leur pertinence pour améliorer les performances du GDA.

\begin{tabular}{|c|c|c|c|c|c|}
\hline Facteurs de performance & $\begin{array}{l}\text { Exemples mis en } \\
\text { discussion avec les } \\
\text { acteurs }\end{array}$ & Agriculteurs & $\begin{array}{l}\text { Personnel } \\
\text { du GDA }\end{array}$ & $\begin{array}{l}\text { Conseil } \\
\text { d'admi-nistra- } \\
\text { tion }\end{array}$ & CRDA \\
\hline \multicolumn{6}{|c|}{ Relation membres - Conseil d'administration } \\
\hline $\begin{array}{l}\text { La capacité du conseil d'admi- } \\
\text { nistration à répondre aux } \\
\text { demandes des membres }\end{array}$ & $\begin{array}{l}\text { La pertinence de } \\
\text { l'acquisition d'un } \\
\text { tracteur par le GDA }\end{array}$ & Favorables & Favorable & Favorable & Opposé \\
\hline $\begin{array}{l}\text { La capacité des membres à } \\
\text { contrôler la gestion du conseil } \\
\text { d'administration }\end{array}$ & $\begin{array}{l}\text { Le processus pertinent } \\
\text { pour empêcher un } \\
\text { endettement futur } \\
\text { du GDA }\end{array}$ & \multicolumn{4}{|c|}{$\begin{array}{l}\text { Meilleure gestion par le CA (mais pas de rôle particulier attribué } \\
\text { aux membres du GDA pour assurer un tel contrôle) }\end{array}$} \\
\hline $\begin{array}{l}\text { L'implication des agriculteurs } \\
\text { dans le fonctionnement du } \\
\text { GDA }\end{array}$ & $\begin{array}{l}\text { La possibilité de faire } \\
\text { participer les agricul- } \\
\text { teurs à l'entretien des } \\
\text { filtres }\end{array}$ & $\begin{array}{l}\text { En majorité } \\
\text { opposés }\end{array}$ & Opposé & Opposé & Favorable \\
\hline \multicolumn{6}{|c|}{ Relation Conseil d'administration - personnel du GDA } \\
\hline $\begin{array}{l}\text { Le pilotage au quotidien par le } \\
\text { conseil d'administration des } \\
\text { activités du personnel } \\
\text { technique }\end{array}$ & $\begin{array}{l}\text { La mise en œuvre d'un } \\
\text { tour d'eau planifié }\end{array}$ & Opposés & Opposé & Favorable & Favorable \\
\hline \multicolumn{6}{|l|}{ Relation GDA - CRDA } \\
\hline $\begin{array}{l}\text { La non-intervention de l'admi- } \\
\text { nistration dans le fonctionne- } \\
\text { ment quotidien de } \\
\text { l'association ET la possibilité } \\
\text { pour l'association de modifier } \\
\text { ses propres limites d'action }\end{array}$ & $\begin{array}{l}\text { Le retrait du CRDA } \\
\text { du contrôle des déci- } \\
\text { sions } \\
\text { du GDA en ce qui } \\
\text { concerne la fourniture } \\
\text { de l'eau hors } \\
\text { périmètre }\end{array}$ & Opposés & Favorable & Opposé & Opposé \\
\hline $\begin{array}{l}\text { L'existence d'un cadre formel } \\
\text { écrit, clair et approuvé par les } \\
\text { parties prenantes, décrivant les } \\
\text { règles de fonctionnement des } \\
\text { relations entre l'association et } \\
\text { l'administration }\end{array}$ & $\begin{array}{l}\text { La possibilité d'inclure } \\
\text { la qualité de l'eau reçue } \\
\text { par le GDA dans le } \\
\text { contrat de gérance } \\
\text { entre GDA et CRDA }\end{array}$ & Favorables & Favorable & Favorable & Opposé \\
\hline
\end{tabular}

La personne du CRDA chargée du suivi des GDA rappelle que le service dans lequel elle travaille n'intervient pas dans la gestion interne du GDA et que, avant la Révolution, le CRDA n'avait pas le pouvoir d'interrompre la fourniture d'eau sans l'accord du ministère de l'Intérieur, ce qui a contribué à l'accumulation des dettes. Cette personne du CRDA considère par ailleurs que les membres du personnel technique ont cherché à conserver leur poste en ne prenant pas position contre la gestion des anciens élus. Tous ces acteurs ont insisté sur l'importance d'une bonne gestion par le CA, mais ils n'ont pas donné d'éléments concrets pour assurer une telle gestion. En particulier, aucun n'a évoqué la nécessité pour les membres du GDA de s'impliquer dans le contrôle de la gestion du CA.

\section{Développement des activités du groupement et participation des membres à la gestion}

Les acteurs du groupement (agriculteurs, CA et service technique) souhaitent que le GDA diversifie ses activités en achetant un tracteur. Cependant, la personne du CRDA ne partage pas leur avis, et rappelle que le GDA ne peut pas avoir une activité à caractère commercial 
autre que la vente d'eau. Cette même personne du CRDA n'écarte pas la possibilité qu'un groupe d'agriculteurs achète un tracteur en commun, mais pas dans le cadre d'un GDA et considère que les priorités du CA et du personnel technique doivent être la gestion de l'eau et l'entretien des équipements du périmètre.

Un des agriculteurs interrogés est d'accord avec l'idée de participer à l'entretien des filtres, et le justifie par l'indisponibilité occasionnelle de l'aiguadier sur le périmètre et par sa charge de travail. Cependant, les autres agriculteurs interrogés pensent que ce n'est pas possible, en raison du nombre réduit d'agriculteurs qui pourraient se porter volontaires. Le CA, quant à lui, justifie son refus d'une telle participation des agriculteurs au fonctionnement du GDA en rappelant que c'est au personnel technique d'assurer la qualité du service et la maintenance. Le personnel technique juge que les agriculteurs n'ont pas les compétences nécessaires pour prendre en charge l'entretien des filtres.

\section{L'autonomie du groupement dans la prise de décision}

La plupart des acteurs interrogés ont exprimé leur intérêt pour que le CRDA contrôle les décisions du CA en ce qui concerne la distribution de l'eau hors du périmètre. Les membres du GDA expriment leur appréhension par rapport au fait de donner de l'eau en dehors du périmètre, car les agriculteurs qui la recevraient pourraient, avec le temps, acquérir un droit d'usage permanent. Un des agriculteurs avance: "Le GDA ne doit prendre aucune décision seul, il faut qu'il avise le CRDA. » Le CA pense qu'il faudrait demander l'avis du CRDA pour que de nouveaux agriculteurs puissent bénéficier de l'eau du périmètre. L'agent CRDA de la cellule de suivi des GDA considère que, pour toutes les sources d'eau utilisées sur le périmètre irrigué, il faudrait respecter les limites définies par l'étude hydraulique initiale. Seul le personnel technique se déclare favorable à une autonomie du GDA : il considère que le GDA devrait avoir plus de marge de manœuvre pour gérer les volumes issus de ses deux forages, de façon à maximiser leur utilisation et ainsi augmenter les revenus tirés de la vente d'eau.

Les agriculteurs ont exprimé leur méfiance vis-à-vis d'un éventuel retrait du CRDA du contrôle de la gestion du GDA. Un agriculteur contacté s'exprime sur ce point : « Je suis contre le retrait du CRDA de la gestion du GDA, il faut qu'il contrôle les débordements qui pourraient arriver. Si le CRDA se retire, le GDA se dissoudra. » Il se demande: "Si le directeur technique et les aiguadiers décident de ne pas travailler, vers qui on va se tourner ? » Le personnel technique partage l'avis des agriculteurs et pense que la présence de l'administration sur le périmètre permet de mieux gérer les problèmes avec les agriculteurs. Le CA ne perçoit pas la présence du CRDA au quotidien du GDA comme une contrainte à sa propre activité de gestionnaire. Ainsi, même si les acteurs du groupement souhaiteraient que le prix de l'eau diminue grâce au retrait du CRDA en tant qu'intermédiaire entre la Secadenord et le GDA, ils voudraient que le CRDA reste présent pour assurer une fonction de contrôle des élus qui pourraient abuser de leur pouvoir. Le CRDA, quant à lui, lie explicitement la question de son contrôle du fonctionnement du GDA à sa position d'intermédiaire pour la distribution de l'eau. Il justifie cette position par les travaux de maintenance du réseau primaire et secondaire qu'il assure sur le périmètre (et dont, selon lui, le GDA sous-estime l'importance). Le CRDA pense que son retrait dans le futur serait cependant envisageable, si le GDA prenait la responsabilité de la gestion du périmètre irrigué tout entier.

\section{Discussion}

\section{Des visions contrastées des objectifs du GDA et des moyens d'améliorer ses performances}

La colonne de droite du tableau 2 permet de récapituler les points de vue des acteurs contactés par rapport à ce qu'ils voudraient que le GDA étudié soit ou fasse, sur la base de la liste d'exemples qui leur est fournie. L'analyse conjointe des différents facteurs permet de mettre en lumière trois enseignements. Premièrement, l'objectif principal du GDA est différemment perçu par le CRDA et par les acteurs du GDA (agriculteurs, personnel technique et CA). L'exemple de l'achat d'un tracteur par le GDA illustre cette différence. Pour les acteurs du groupement, le GDA devrait avant tout répondre aux demandes des membres, tandis que pour l'administration, le GDA doit rester une structure de gestion de l'eau. Cette mission de maintenance de l'infrastructure hydraulique doit, selon le CRDA, être prioritaire sur celle de l'accompagnement d'une dynamique collective.

Deuxièmement, chaque catégorie d'acteurs rejette sur d'autres la responsabilité d'améliorer les performances. Ceci est illustré par la question de la qualité de l'eau, traitée à travers deux exemples : l'implication des agriculteurs dans la maintenance au quotidien des filtres et l'intégration de la qualité de l'eau dans le contrat de gérance CRDA-GDA. Les positions des acteurs interrogés sont semblables dans les deux cas. Les acteurs du groupement sont favorables à l'introduction de la qualité de l'eau dans le contrat de gérance et sont opposés à la participation des agriculteurs à la maintenance des filtres. De son côté, le CRDA est enthousiaste à l'idée que les agriculteurs soient actifs dans le nettoyage des filtres, mais refuse l'inclusion de la qualité de l'eau dans le contrat de 
gérance, arguant que cette qualité ne dépend pas de lui, mais de la Secadenord en amont. Ces deux exemples montrent que les deux groupes d'acteurs (GDA d'un côté et CRDA de l'autre) se rejettent mutuellement la responsabilité de l'amélioration de la qualité de l'eau fournie aux agriculteurs. De même, les agriculteurs ne s'estiment pas « parties prenantes » de la question du contrôle du CA, en particulier de sa bonne gestion.

Troisièmement, l'autonomie du groupement vis-àvis du CRDA n'est pas recherchée par les acteurs du GDA, à l'exception du personnel technique. Un cercle vicieux s'est établi : les agriculteurs estiment n'avoir que peu d'emprise sur le CA et, de ce fait, souhaitent un contrôle par le CRDA. Cependant, la présence du CRDA contribue à son tour à affaiblir la relation entre agriculteurs et $\mathrm{CA}$; les agriculteurs se sentent moins responsables du fonctionnement du GDA et le CA estime que les agriculteurs ne sont pas les seuls à qui il doit rendre des comptes. Les agriculteurs sont, par manque de confiance envers le CA, en demande d'une présence permanente de l'administration comme garantie de la bonne gestion du GDA. Ce cercle vicieux est relativement stable car il n'y a pas de «crise »: dans l'ensemble, le GDA assure correctement ses fonctions de base de distribution de l'eau et de facturation, malgré l'absence d'une réelle intervention du CA dans la gestion quotidienne. Le personnel technique souhaite conserver voire augmenter son autonomie, ceci à la fois vis-à-vis du CRDA pour ce qui concerne la vente d'eau hors du périmètre et du CA pour ce qui concerne la gestion du tour d'eau.

Les acteurs du GDA étudié sont dans l'ensemble passifs vis-à-vis de l'amélioration des performances. Les discours pour expliquer cette passivité renvoient à des contraintes objectives. Ainsi, le CA et le personnel technique déclarent que l'endettement du GDA les empêche de prendre des initiatives. Cependant, les trois éléments exposés ci-dessus mettent en valeur la forte différence de conception des différents acteurs sur les objectifs du GDA, et les moyens pour les atteindre; ceci empêche aussi leur prise d'initiative. De plus, il n'y a pas de « coalition » des quatre groupes d'acteurs (agriculteurs, élus $\mathrm{du}$ CA, personnel technique, CRDA) pour exprimer un point de vue commun sur le rôle du GDA et les moyens qu'il doit mettre en œuvre : les différences entre les acteurs favorables et ceux opposés à telle ou telle option varient d'un facteur à l'autre.

\section{Retour sur la méthode}

La phase exploratoire a permis de discuter avec les acteurs des objectifs et des missions du GDA et de l'administration. Cette première phase a révélé que les acteurs définissent les missions actuelles du GDA comme étant la fourniture d'eau et la maintenance des infrastructures hydrauliques. Ils pensent que, pour qu'il soit performant, le GDA doit fournir un service flexible, assurer une situation financière stable et répondre au mieux aux besoins des agriculteurs.

La deuxième phase de mise en débat des facteurs de performance, tirés des grilles d'analyse, a pu être faite grâce à la connaissance fine du GDA permise par la phase exploratoire. Ceci a conduit parfois à choisir des propositions qui ne semblaient pas, de prime abord, explicitement liées à ces grilles. Ce fut le cas pour étudier la pertinence de la flexibilité du tour d'eau, exemple que nous avons jugé approprié pour rendre compte de la capacité du CA à bien diriger le service technique.

Une mise en discussion des facteurs de performance de leur association aurait bien sûr été possible avec les acteurs sans avoir recours aux théories de l'action collective. Cette utilisation, dans notre étude, a cependant pu apporter une forte valeur ajoutée, pour rendre plus explicite la diversité des définitions des performances du GDA et pour identifier les points de blocage qui conditionnent leur amélioration.

Les différentes étapes de la méthode ont bien fonctionné ici. Il est cependant possible que, dans d'autres cas, il ne soit pas aisé d'identifier dans la littérature des principes qui correspondent à des enjeux identifiés localement. Si ce type de situation est a priori peu fréquent (les trois grilles d'analyse utilisées ici et les principes de bonne gestion correspondants ayant été construits à partir d'un éventail très large de situations), ceci montre l'intérêt de tester une telle démarche dans d'autres situations d'action collective.

\section{Conclusion}

La méthode utilisée atteste, à travers l'étude d'un groupement d'irrigants, de la pertinence des théories de l'action collective pour accompagner les acteurs dans l'explicitation de leur vision du fonctionnement de leur organisation et des facteurs qui influencent ses performances.

À la différence d'une approche d'évaluation «classique », nous n'avons pas cherché à établir un diagnostic de performance du groupement, en analysant si tel ou tel facteur de performance proposé par différentes théories sur l'action collective était présent ou non. Nous avons utilisé ces facteurs pour structurer une vision globale à partir des points de convergence et de divergence des opinions des acteurs sur le fonctionnement souhaité et actuel de leur GDA. Il s'agissait ainsi de mieux comprendre la complexité des relations entre les différents acteurs impliqués dans ce groupement.

L'analyse des interprétations qu'ont les acteurs de leur propre action collective a montré l'importance aussi 
bien du contexte de gestion de leur GDA que des relations qu'entretient chacun d'eux avec les différentes parties prenantes, qui orientent à la fois la gestion actuelle du groupement et les négociations autour des enjeux de sa gestion future. Les trois grilles d'analyse mobilisées portent cependant sur l'évaluation d'une situation à un moment donné. Or, les associations d'irrigants évoluent de façon permanente, et en particulier en fonction des changements qui interviennent au niveau national tels que la révolution de 2011 en Tunisie. Il serait donc intéressant de mieux prendre en compte cet aspect dynamique, et notamment l'évolution des capacités d'analyse des acteurs par l'apprentissage (Argyris et Schön, 2002), que celui-ci soit lié à une formation ou à l'expérience, et l'impact de cet apprentissage sur la transformation des attentes des acteurs par rapport à leur organisation collective et ses performances.

\section{Remerciements}

Nous tenons à remercier l'ensemble des acteurs ayant participé à l'enquête pour leur disponibilité et leur appui. Les opinions exprimées sont de la seule responsabilité des auteurs.

\section{Références}

Al Atiri, R., 2007. Évolution institutionnelle et réglementaire de la gestion de l'eau en Tunisie: vers une participation accrue des usagers de l'eau, in Bouarfa, S., Kuper, M., Debbarh, A. (Eds), L'avenir de l'agriculture irriguée en Méditerranée. Nouveaux arrangements institutionnels pour une gestion de la demande en eau, Actes du séminaire Wademed, 6-7 novembre 2006, Montpellier, Cirad (online: http:// hal.archives-ouvertes.fr/docs/00/19/10/75/PDF/IAl_Atiri.pdf).

Argyris, C., Schön, D.A., 2002. Apprentissage organisationnel. Théorie, méthode, pratique (M. Aussanaire et P. GarciaMelgares, trad.), Bruxelles, De Boeck Université.

Ben Salem, H., Zaibet, L., Bachta, M.S., 2007. Formation des revenus des exploitants, in Bachta, M.S. (Ed.), Les instruments économiques et la modernisation des périmètres irrigués, Cirad, Kairouan, Tunisie (online: http:// hal.archives-ouvertes.fr/cirad-00193848/).

Beaurain, C., Longuépée, J., Soussi, S.P., 2009. La proximité institutionnelle, condition à la reconquête de la qualité de l'environnement. L'exemple de l'agglomération dunkerquoise, Natures Sciences Sociétés, 17, 4, 373-380.

Bos, M.G., Murray-Rust, D.H., Merrey, D.J., Johnson, H.G., Snellen, W.B., 1994. Methodologies for assessing performance of irrigation and drainage management, Irrigation and Drainage Systems, 7, 231-261.

Canesse, A.A., 2010. Les groupements de développement agricole en Tunisie: entrepreneurs locaux ou relais administratifs ?, in Denieuil, P.N., Madoui, M. (Eds), Entrepreneurs maghrébins, terrains en développement, Paris, Karthala, 243-255.

Elloumi, M., 2011. Pour une gestion durable des ressources naturelles, les limites du cadre institutionnel tunisien, in Dahou, T., Elloumi, M., Molle, F., Gassab, M., Romagny, B. (Eds), Pouvoirs, sociétés et nature au sud de la Méditerranée, Paris, Karthala, 53-80.

Elloumi, M., Sghaier, M., Dhehibi, B., Kadhkadhi, K., 2010. Changement institutionnel et développement durable : vers une nouvelle gouvernance du développement local et de la gestion des ressources naturelles, in Bonte, P., Elloumi, M., Guillaume, H., Mahdi, M. (Eds), Développement rural, environnement et enjeux territoriaux: regards croisés Oriental marocain et Sud-Est tunisien, Tunis, Cérès, 199-222.

Gana, A., 2011. Agriculteurs et paysans, nouveaux acteurs de la société civile et de la transition démocratique en Tunisie, Observatoire Tunisien de la Transition Démocratique, Tunis, Éditions Diwan.

Giddens, A., 1984. The Constitution of Society: Outline of the Theory of Structuration, Berkley (CA), University of California Press.

Giordano, M.A., Samad, M., Namara, R.E., 2006. Assessing the Outcomes of IWMI's Research and Interventions on Irrigation Management Transfert. IWMI Research Report 106, International Water Management Institute, Colombo, Sri Lanka.

Johnson, S.H., Vermillion, D.L., Sagardoy, J.A., 1995. Irrigation management transfer: Selected papers from the international conference on irrigation management transfer. Water Report 5, Wuhan, China, September 20-24, 1994, Rome, FAO and IIMI.

Meinzen-Dick, R., DiGregorio, M., McCarthy, N., 2004. Methods for studying collective action in rural development, Agricultural Systems, 82, 3, 197-214.

Mermet, L., Billé, R., Leroy, M., Narcy, J.B., Poux, X., 2005. L'analyse stratégique de la gestion environnementale : un cadre théorique pour penser l'efficacité en matière d'environnement, Natures Sciences Sociétés, 13, 2, 127-137.

Mouri, H., Marlet, S., 2006. De l'association d'intérêt collectif au groupement de développement agricole : le changement institutionnel et son impact sur le fonctionnement des périmètres publics irrigués tunisiens, Actes du séminaire Wademed, 67 novembre 2006, Montpellier, Cirad (online: http:// hal.ird.fr/file/index/docid/191058/filename/IMourri_et_Marlet.pdf).

Ostrom, E., 1990. Governing the Commons: The Evolution of Institutions for Collective Action, Cambridge, Cambridge University Press.

Ostrom, E., 1992. Crafting Institutions for Self-Governing Irrigation Systems, San Francisco, Institute for Contemporary Studies.

Ostrom, E., 2000. Reformulating the commons, Swiss Political Science Review, 6, 1, 29-52.

Romagny, B., Riaux, J., 2007. La gestion communautaire de l'eau agricole à l'épreuve des politiques participatives: regards croisés Tunisie/Maroc/Community-based agricultural water management in the light of participative policies : A cross-cultural look at cases in Tunisia and Morocco, 
Hydrological Sciences Journal/Journal des Sciences Hydrologiques, 52, 6, 1179-1196.

Samad, M., Vermillion, D., 1999. An assessment of the impact of participatory irrigation management in Sri Lanka, International Journal of Water Resources Development, 15, 1-2, 219-240.

Shah, T., 1995. Making Farmers Cooperatives Work: Design, Governance and Management, New Delhi, India, Sage Publications.
Shah, T., 1996. Catalysing Co-Operation Design of Self-Govering Organisations, New Delhi, India, Sage Publications.

Shah, T., Van Koppen, B., Merrey, D., De Lange, M., Samad, M., 2002. Institutional Alternatives in African Smallholder Irrigation. Research Report 60, International Water Management Institute, Colombo, Sri Lanka.

Vermillion, D.L., 1997. Impacts of Irrigation Management Transfer: A Review of the Evidence. Research Report 11, International Irrigation Management Institute, Colombo, Sri Lanka. 